\title{
Vertical and Horizontal Ridge Augmentation of a Severely Resorbed Ridge in the Anterior Maxilla
}

\author{
Alberto Monje, ${ }^{\star}$ Florencio Monje, ${ }^{\dagger}$ Fernando Suarez, ${ }^{*}$ Raúl González-García, ${ }^{\dagger}$ Laura Villanueva-Alcojol, ${ }^{\dagger}$ Carlos Moreno, ${ }^{\dagger}$ \\ Pablo Galindo-Moreno, ${ }^{\ddagger}$ and Hom-Lay Wang*
}
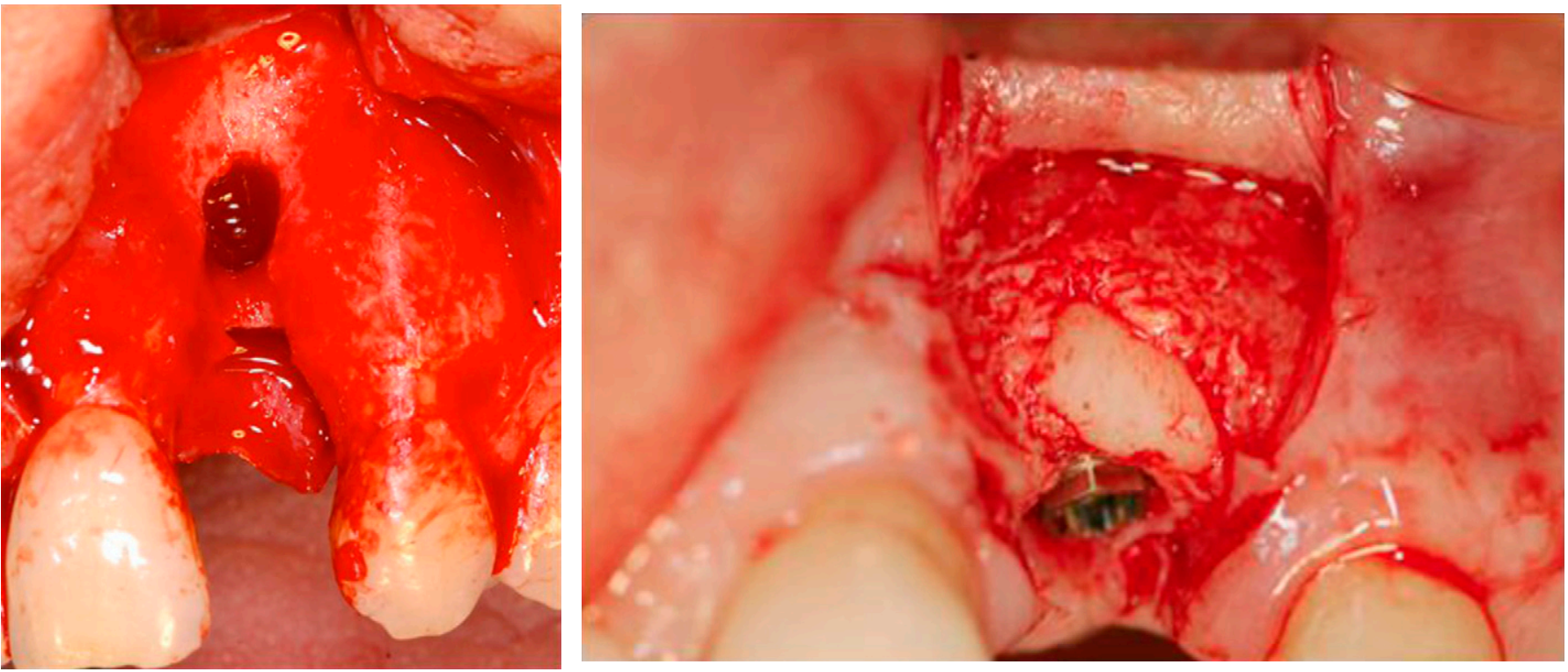

Introduction: Achieving esthetically pleasing therapeutic outcomes when rehabilitating a deficient anterior maxilla with dental implants can be very challenging. This case report presents the use of platelet-rich plasma (PRP) and guided bone regeneration (GBR) in combination with autogenous block grafts in the three-dimensional bone augmentation of a severely deficient anterior maxillary ridge.

Case Presentation: A patient with a missing maxillary left lateral incisor received a cone-beam computed tomography scan, which revealed a horizontally and vertically deficient residual ridge. Horizontally, the residual had no buccal plate and a thin palatal plate. Vertically, the defect was $14.5 \mathrm{~mm}$ as measured from the cemento-enamel junction of adjacent teeth to the most apical point. Both buccal and palatal sites were treated with autogenous ramus block grafts, GBR, and PRP in an attempt to gain adequate ridge height and width for future implant placement. The surgical site was reentered 6 to 9 months after the bone augmentation procedure, and a dental implant, with no additional bone grafting, was placed with adequate primary implant stability. As a result of the successful gain in bone width and height through the augmentation procedure, an esthetic outcome was achieved with the implant-supported restoration. In addition, no signs of inflammation, peri-implant bone loss, or implant mobility were observed.

Conclusion: Vertical and horizontal bone augmentation can be successfully performed to gain bone height and width that is essential for ideal implant positioning and esthetic outcomes. Clin Adv Periodontics 2013;3:230-236.

Key Words: Alveolar ridge augmentation; bone transplantation; dental implants, single-tooth.

\footnotetext{
* Department of Periodontics and Oral Medicine, University of Michigan, School of Dentistry, Ann Arbor, MI.

† Department of Oral and Maxillofacial Surgery, Center of Implantology, Oral and Maxillofacial Surgery, Badajoz, Spain.

${ }^{\ddagger}$ Department of Oral Surgery, University of Granada, Granada, Spain.
}

\section{Background}

One of the most important factors that influence the esthetic therapeutic outcome of implant treatment is the presence of the buccal alveolar bone. ${ }^{1}$ Periodontal disease, traumatic extractions, aberrant bony tomography, and physiologic resorption attributable to tooth extraction can lead to vertical and horizontal alveolar bone loss, ${ }^{2}$ adversely compromising implant treatment. ${ }^{1}$ Generally, as a result of the bone loss volume, it is a challenge to place the implant in a proper three-dimensional position, 


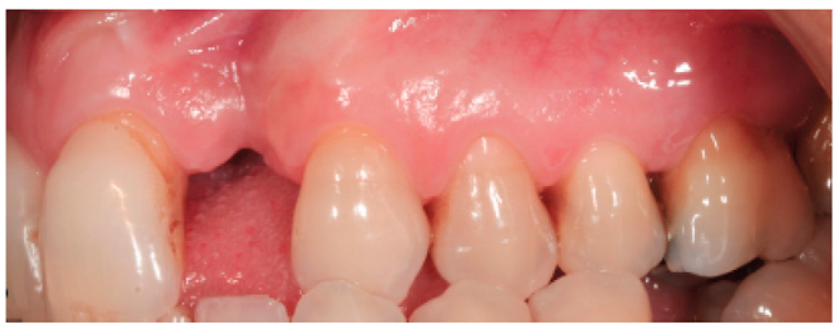

FIGURE 1 Initial clinical view of the deficiency. A slight vertical defect is observed, indicating an esthetic challenge from the restorative perspective.
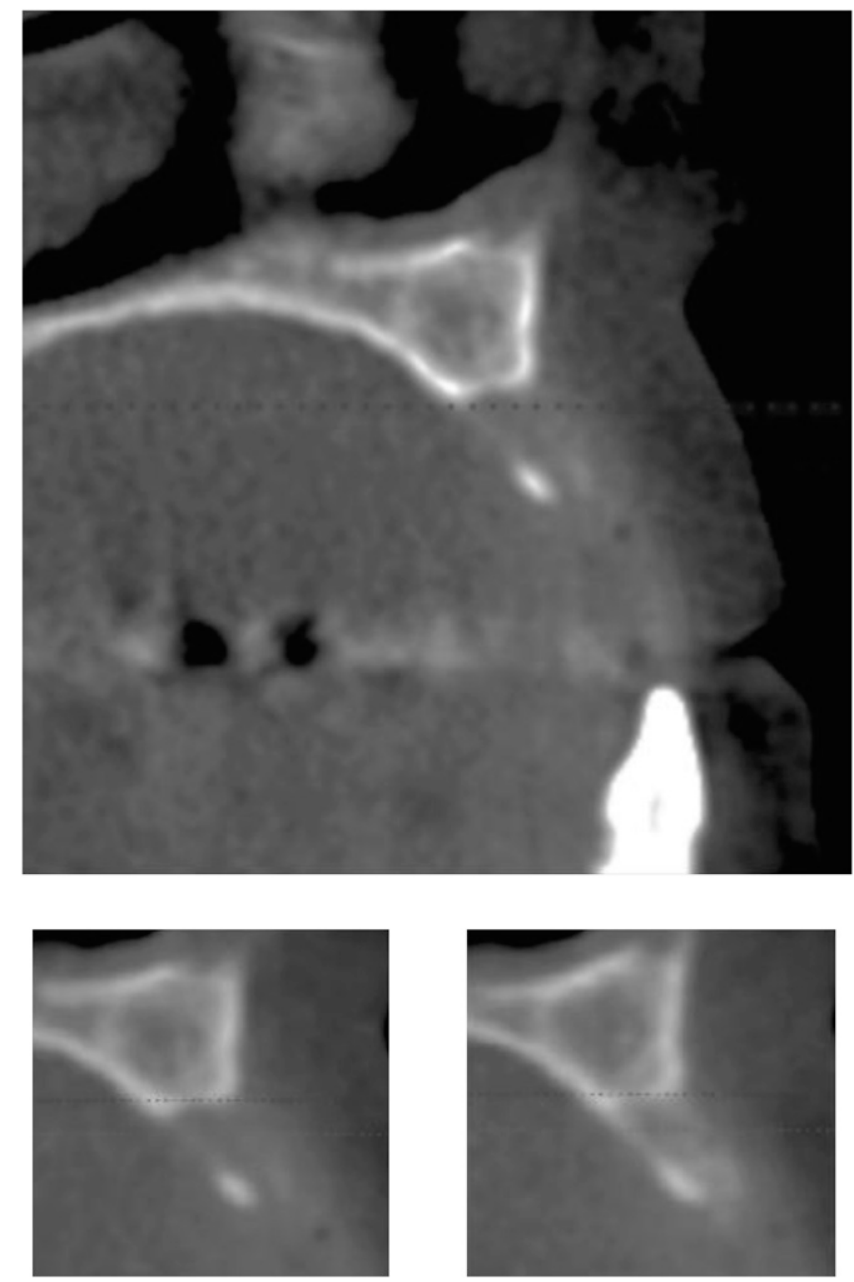

FIGURE 2 Preoperative sagittal views of the defect. The buccal plate was absent, and only a thin palatal plate was present.

without which, long-term implant stability and esthetic outcomes are compromised. Hence, to achieve a successful functional and esthetic implant-supported restoration, atrophic ridges often require hard-tissue and/or soft-tissue grafting before implant placement.

Autogenous block graft is considered the gold standard in ridge augmentation procedures because of its osteogenic, osteoinductive, and osteoconductive properties. Two common intraoral donor sites are the mandibular ramus and symphysis. Intraoral autogenous grafts have several benefits, such as less bone resorption after healing
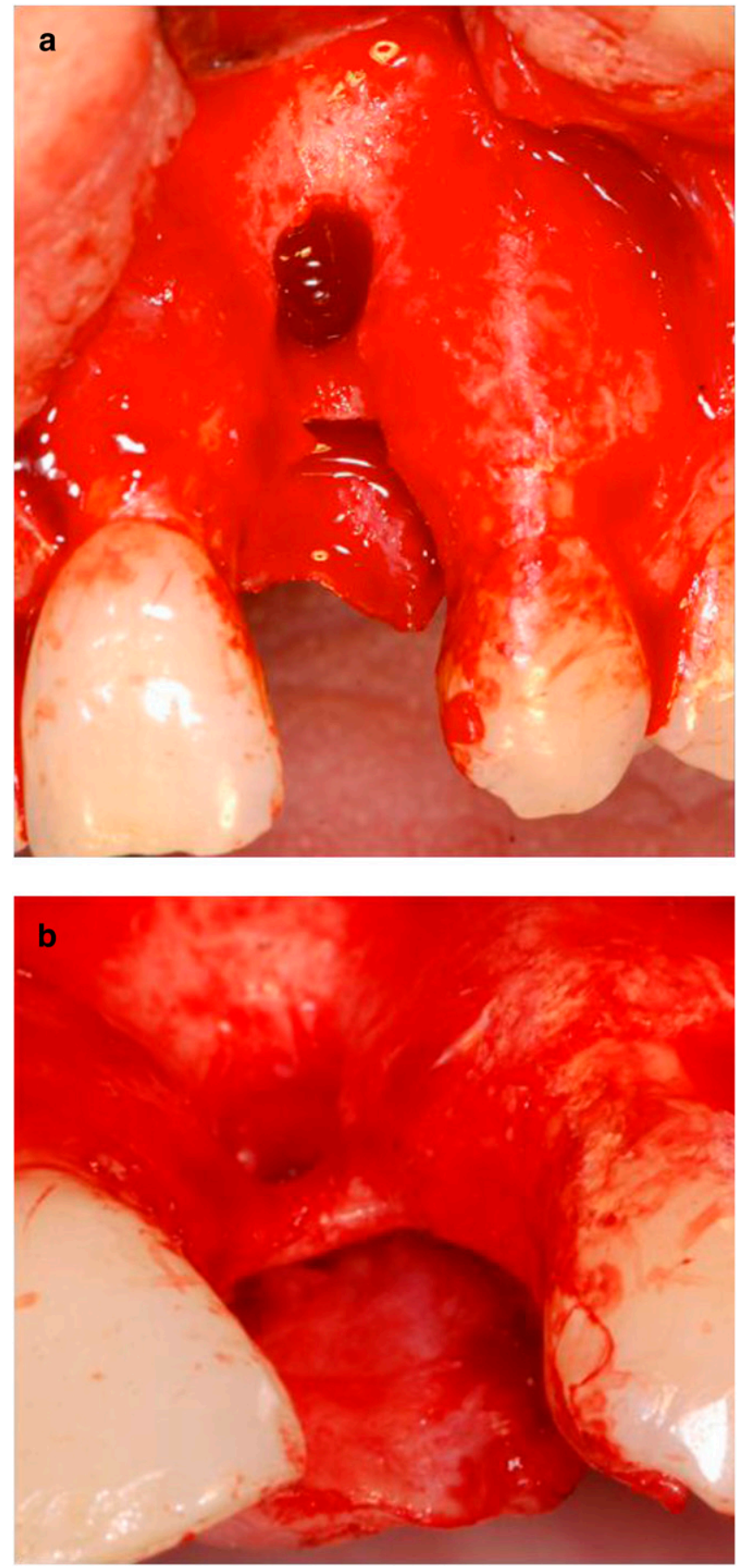

FIGURE 3 Clinical views of the hard-tissue defect. 3a Intraoral frontal view. 3b Intraoral occlusal view.

when compared with extraoral sites (e.g., tibia, iliac crest), ${ }^{3}$ and graft harvesting can be performed under local anesthesia. ${ }^{4}$ Conversely, limitations are donor-site morbidity and limited availability. Techniques, such as distraction osteogenesis, guided bone regeneration (GBR) using particulate bone, and allogenic or autogenous block grafts, have also been proposed for both horizontal and vertical bone augmentation. Although horizontal bone augmentation is reasonably predictable, vertical bone augmentation using onlay block grafts remains unpredictable. ${ }^{5}$ This is because of the inherent complexity to gain revascularization of the entire graft and the lack 


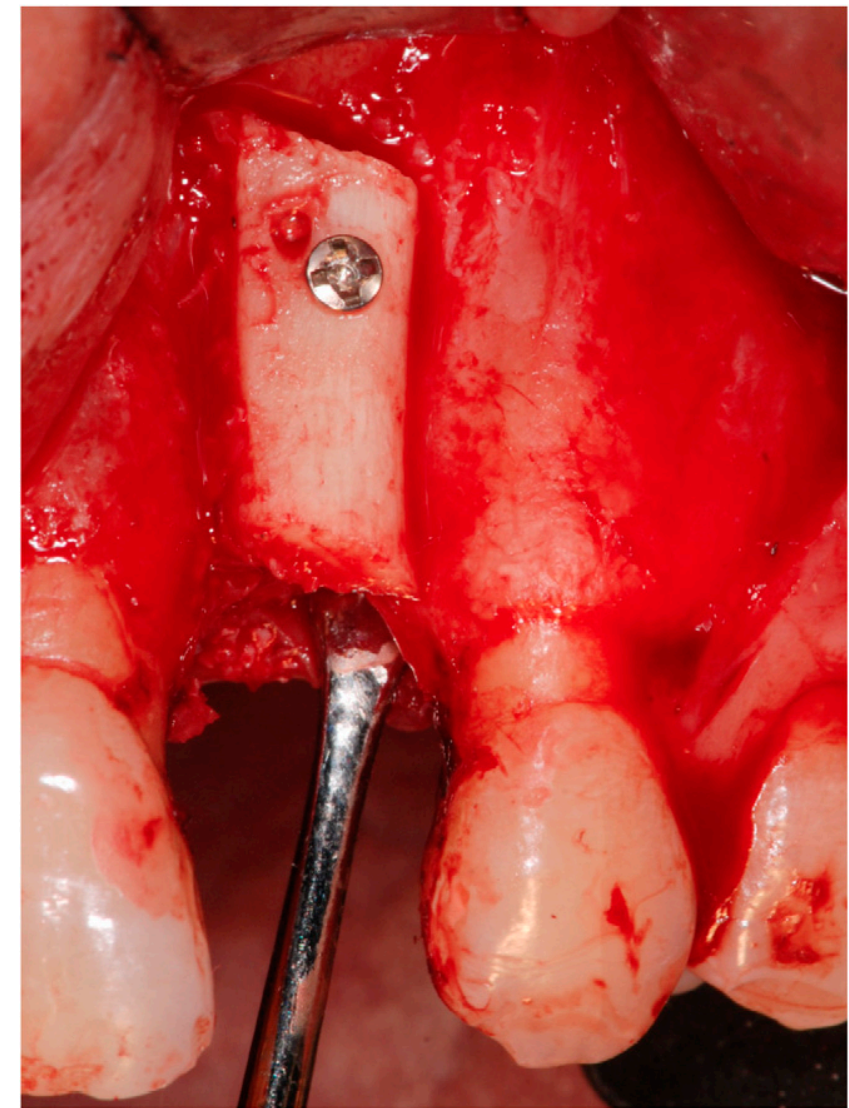

FIGURE 4 Adaptation of one of the monocortical grafts to the recipient site anchored to the residual ridge by two $1.5-\mathrm{mm}$ titanium fixation screws.

of primary wound closure as a result of insufficient soft tissue. The purpose of this case report is to describe the use of autogenous block grafts, GBR, and plateletrich plasma (PRP) in vertical and horizontal bone augmentation of an atrophic maxillary anterior residual ridge.

\section{Clinical Presentation}

A 47-year-old white female presented to the Center of Implantology, Oral and Maxillofacial Surgery in Badajoz, Spain in November 2009 for fixed prosthetic rehabilitation of her missing left maxillary lateral incisor. The patient reported that the tooth was extracted 1 year previously as a result of pain, mobility, and bone loss and socket preservation was performed after the extraction. However, the patient had a postoperative infection that resulted in the removal of the socket graft. The patient was a healthy nonsmoker with good hygiene. The clinical examination showed that the residual had sufficient vertical bone height but inadequate horizontal bone width (Fig. 1), but the cone-beam computed tomography scan ${ }^{\S}$ revealed the absence of a buccal plate, and only a thin cortical palatal plate was present (Fig. 2). The vertical bone defect as measured from the cemento-enamel junction of adjacent teeth to the most apical point was $14.5 \mathrm{~mm}$. As such, the use of an autogenous ramus graft for horizontal and vertical bone augmentation was proposed and accepted in the written informed consent received by the patient before implant placement.

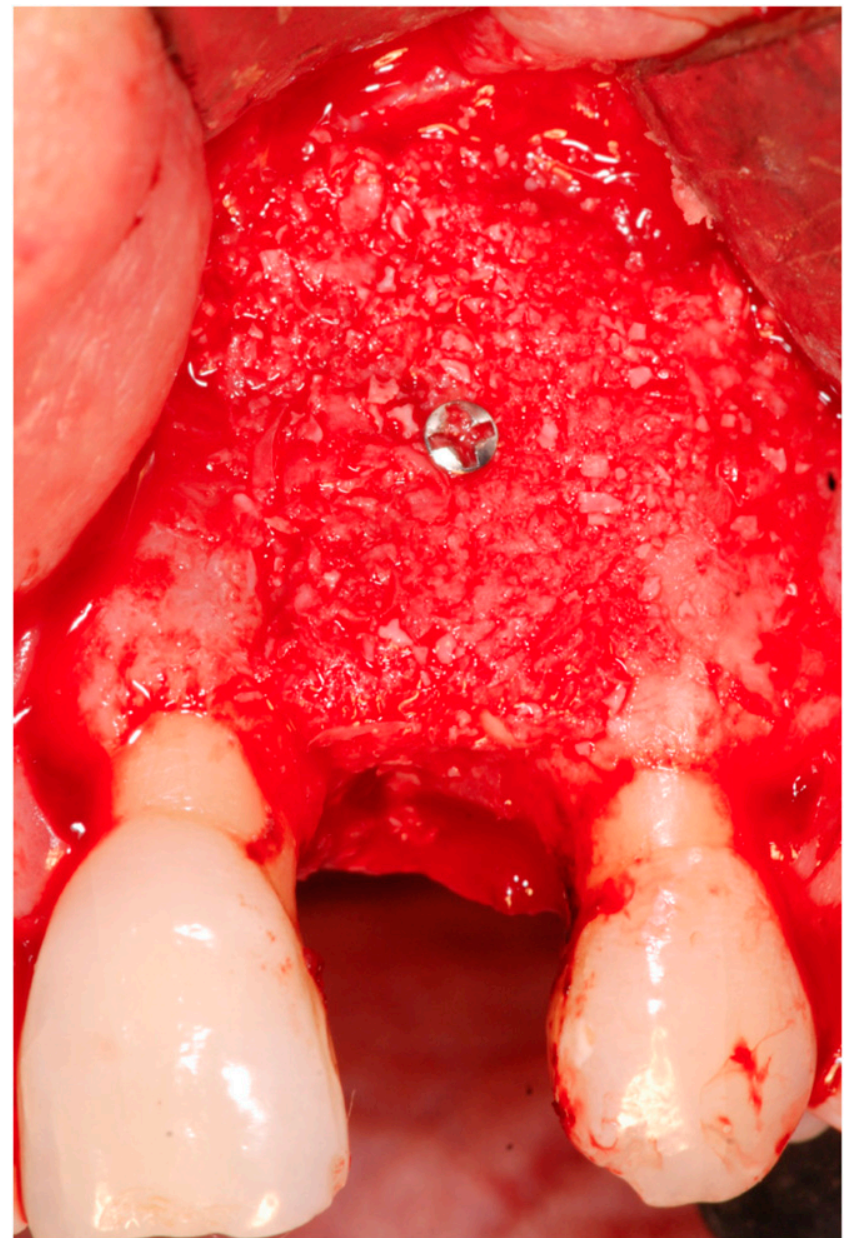

FIGURE 5 Placement of particulated xenograft between and around the monocortical grafts.

\section{Case Management}

Under local anesthesia with intravenous sedation, an incision was performed in the posterior mandible following the external oblique line in the right mandible. A full-thickness flap was reflected exposing the lateral aspect of the ramus. Two rectangular-shaped grafts of $15 \times$ $7.9 \mathrm{~mm}$ were harvested. At the recipient site, a midcrestal incision was performed in the left maxillary lateral incisor area with intrasulcular and vertical releasing incisions on the adjacent teeth. A full-thickness flap was reflected to expose the defect (Fig. 3). Both ramus block grafts were adapted to the recipient sites (buccal and palatal bone) and anchored to the alveolar bone by two 1.5$\mathrm{mm}$ diameter titanium fixation screws" (Fig. 4). After achieving stability of both grafts, sharp edges of grafts were smoothened using a fissure bur. Deproteinized particulated bone grafts were packed around both buccal and palatal ramus grafts to fill any voids (Fig. 5). A collagen absorbable membrane was then placed over both buccal and palatal grafts to promote osseous regeneration (Fig. 6). PRP was harvested and placed over the membrane to promote soft-tissue healing (Fig. 7). Periosteal flap

$\S$ i-CAT, Imaging Sciences International, Hatfield, PA.

" Level One 1.5 Neuro System, KLS Martin, Jacksonville, FL. 


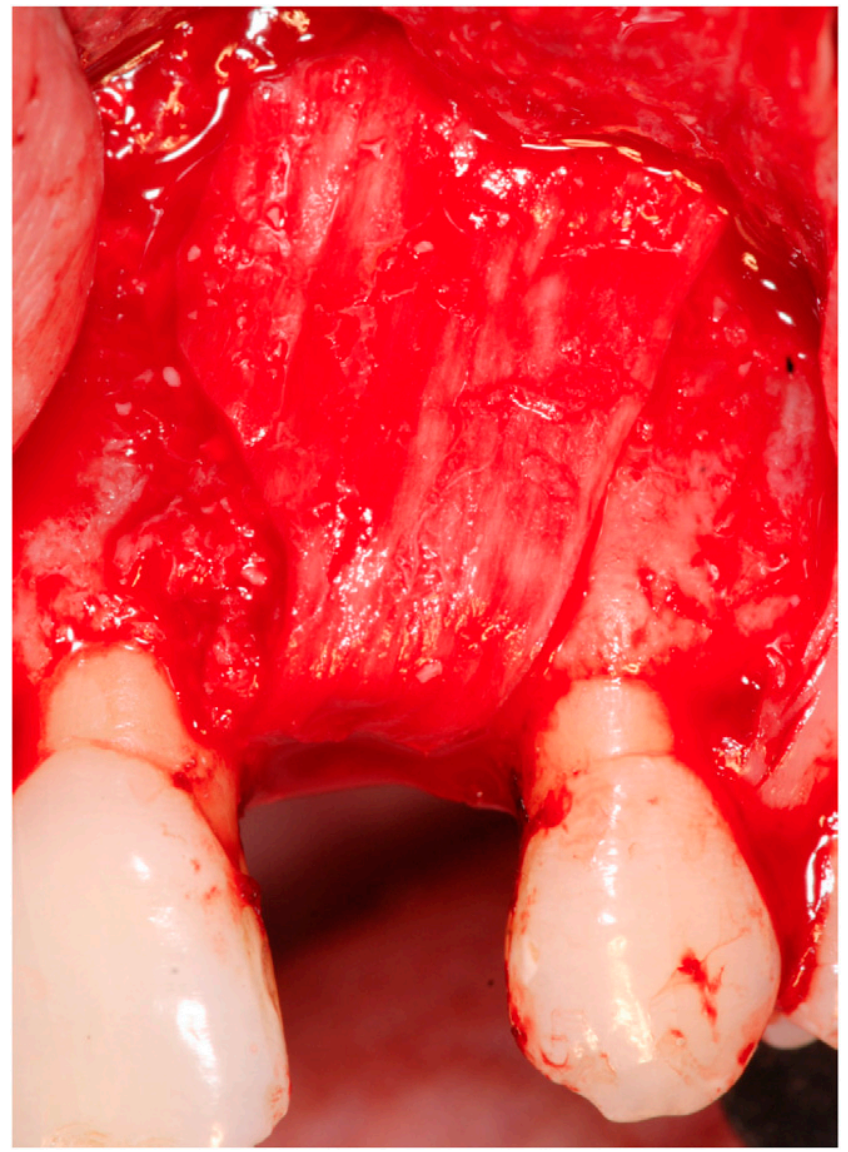

FIGURE 6 Absorbable membrane placed over the particles to promote bone regeneration.

release was performed to achieve tension-free primary wound closure. The donor and recipient sites were subsequently closed with both resorbable and non-resorbable sutures. At the 2-week postoperative review, the recipient site had a wound-healing inde $x^{6}$ score of 2 , which indicated slight gingival edema, erythema, and patient discomfort. Healing of the recipient site slowly improved over time without any complications (Fig. 8). Radiographic bone formation was observed at the 8-month postoperative review (Fig. 9).

Implant placement was performed under local anesthesia. During flap reflection, bone formation was observed at the surgical site. One $3.75 \times 11.5 \mathrm{~mm}$ implant" ${ }^{\#}$ was placed with good implant stability** (implant stability quotient [ISQ] $=$ 74) (Fig. 10). Three months later, a healing abutment was placed with ISQ $=82$ (Fig. 11). A final ceramic-metal screw-retained crown was installed 6 months after implant placement (Figs. 12a and 12b).

\section{Clinical Outcomes}

After 1 year, no peri-implant inflammation and/or bone loss or implant mobility were reported. In addition, the patient was very pleased with the esthetic outcome (Fig. 12c).

\section{Discussion}

Augmentation is often required to achieve a satisfactory esthetic outcome with dental implants in a deficient anterior maxilla. Autogenous bone is the gold standard for

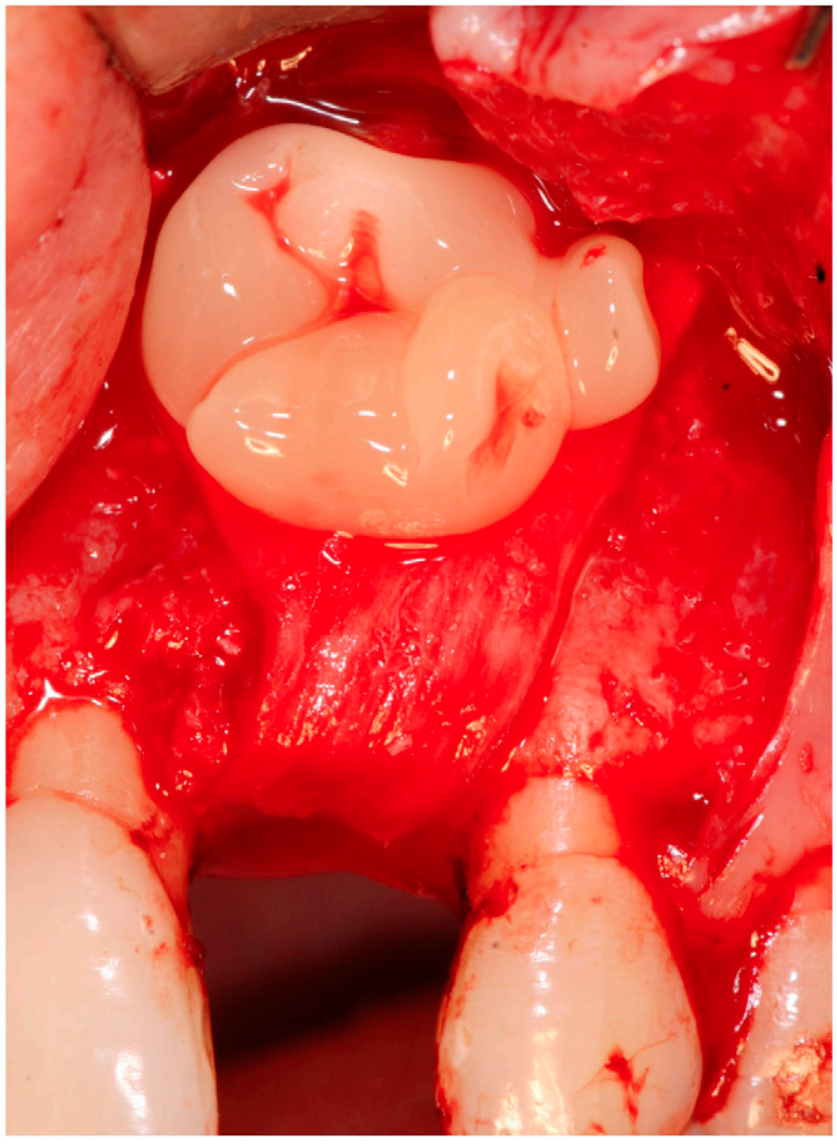

FIGURE 7 PRP placed over the membrane to promote soft-tissue healing.

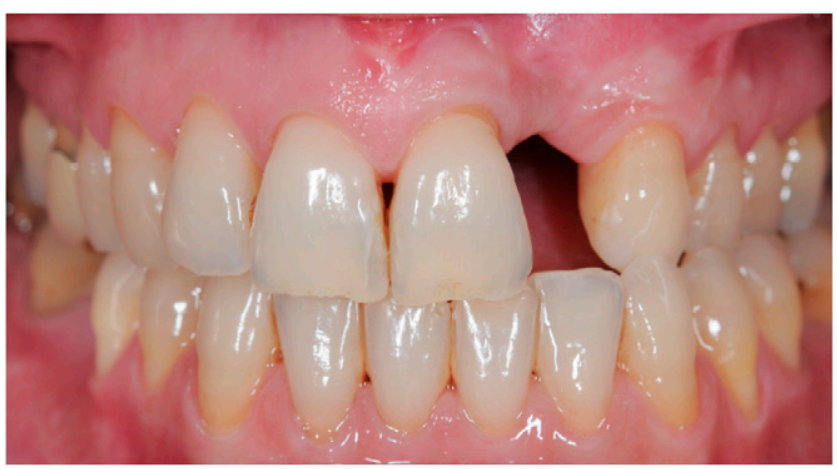

FIGURE 8 Extraoral view of the grafted area 8 months after bone augmentation.

bone augmentation because of its osteogenic ability. Because intraoral grafts have a lesser resorption, higher revascularization, and better graft incorporation compared with extraoral grafts, ${ }^{3}$ they are the best material available to reconstruct isolated horizontal and vertical defects. Symphyseal and ramus block grafts are the most common intraoral donor sites. Although symphyseal block grafts provide a thicker graft, ramus grafts have been shown to have a lower incidence of postoperative sensory disturbances. ${ }^{4} \mathrm{In}$

\footnotetext{
- Cytoplast Suture, Osteogenics Biomedical, Lubbock, TX.

\# NobelSpeedy Groovy Implants, Nobel Biocare, Gothenburg, Sweden.

**Osstell, Gothenburg, Sweden.
} 

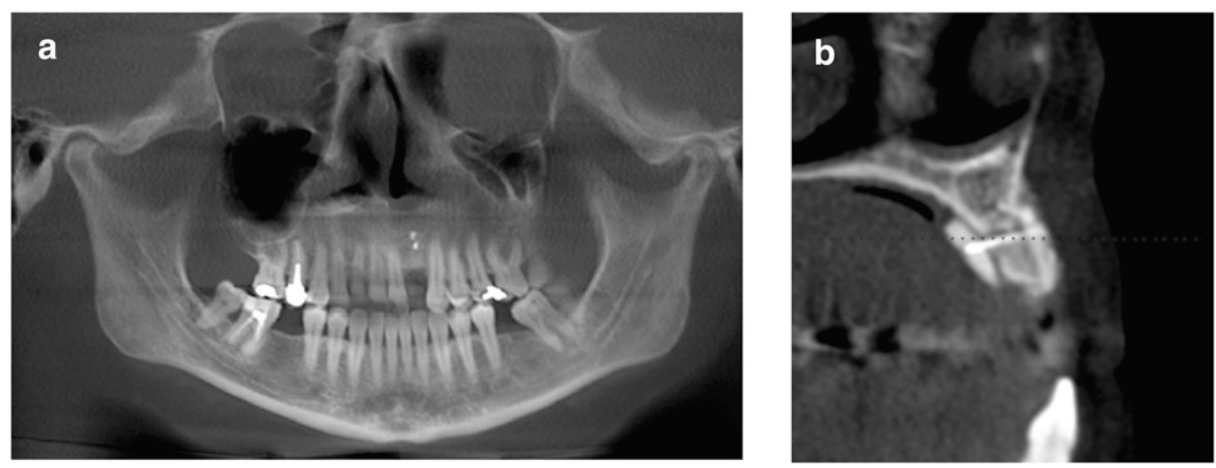

FIGURE 9 Postoperative radiographs. 9a Panoramic radiograph. $9 \mathrm{~b}$ Sagittal view of the grafted area. Grafts were anchored to each other and the recipient site by the fixation screws.
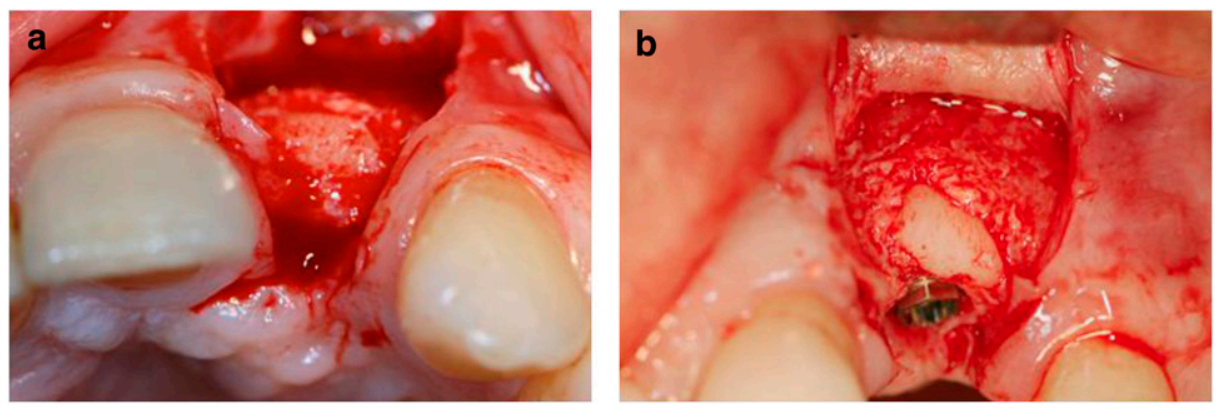

FIGURE 10 Implant placement surgery 8 months after bone augmentation. 10a Intraoral occlusal view of ridge before implant placement. $10 \mathrm{~b}$ Intraoral frontal view of implant placed.

addition, ramus grafts have a higher acceptance rate than symphyseal grafts. ${ }^{4}$ Furthermore, the bone substitute around the block graft used in treating this patient provided the space maintenance for bone to ingrowth. ${ }^{7}$

Barrier membranes were generally used to exclude unwanted cells (fibroblasts) from populating the surgical site, thus promoting bone regeneration. Non-resorbable barrier membranes provide the grafted area with more isolation, rigidity, and protection from mechanical forces. However, non-resorbable membranes have shown higher membrane exposure rate associated with higher incidence of infection, incomplete healing, and connective tissue formation below the membrane as a result of the micromovement of the barrier. ${ }^{8}$ On the contrary, collagen absorbable membranes have some biologic advantages, such as the higher stimulation of deoxyribonucleic acid synthesis over non-resorbable membranes. ${ }^{9}$ It seems that barrier membranes are not required when block grafts are used in ridge augmentation because of the space-maintaining property of the graft. ${ }^{10}$ However, a collagen membrane was used in this case to contain and protect the particulated graft particles placed around the block graft. Also, the membrane could potentially retard graft resorption.

PRP is an autologous platelet concentrate suspended in plasma. These platelets contain growth factors, such as epidermal growth factor, vascular endothelial growth factor, transforming growth factor- $\beta 1$, and platelet-derived growth factor- $\mathrm{BB}$, which initiate and modulate both soft- and hard-tissue wound healing. ${ }^{11}$ Although PRP is often used to manage chronic non-healing wounds, it is also used in oral and maxillofacial surgery to enhance wound healing and bone growth. ${ }^{12}$ A review of the literature concerning PRP and its use for hard- and soft-tissue
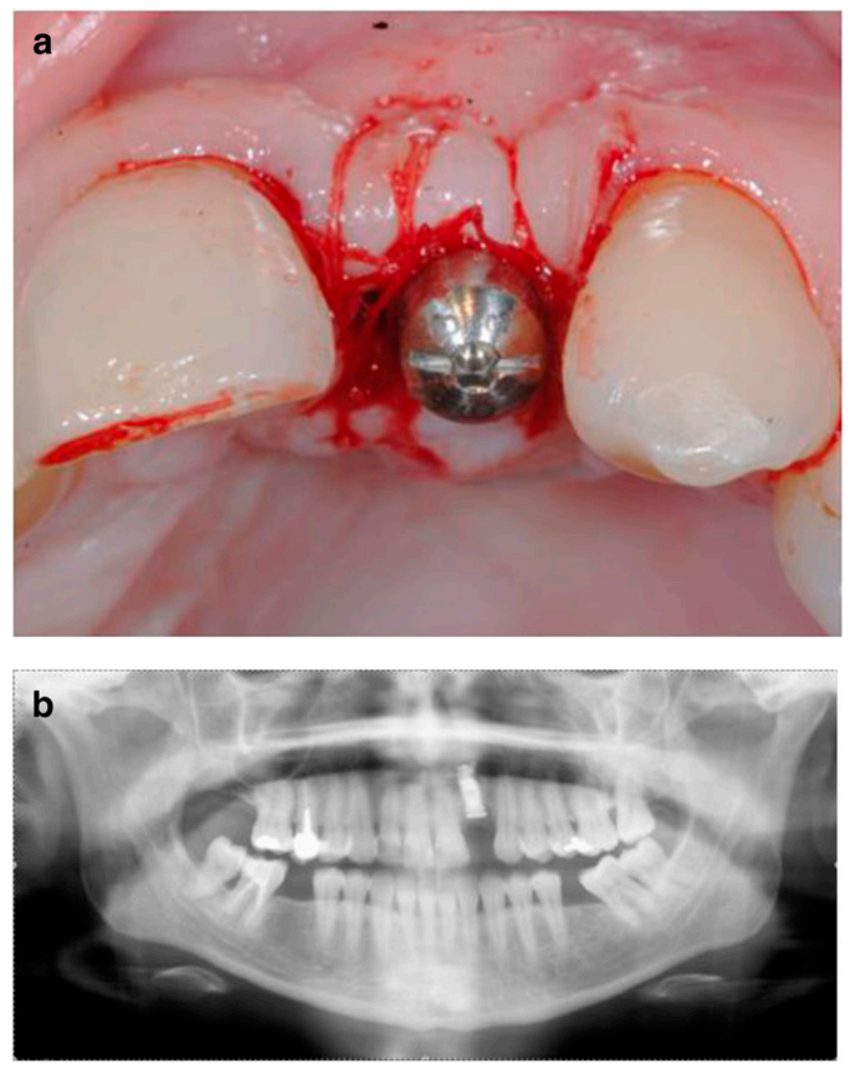

FIGURE 11 Abutment placement 3 months after implant placement. 11a Coronal intraoral view of the abutment placed. 11b Radiographic view of the implant placed just before abutment placement.

healing concluded that, despite the great variability in study designs, autologous platelets constitute a safe, reproducible, and effective means of reproducing the process of wound healing. ${ }^{12}$ The rationale of adding PRP 

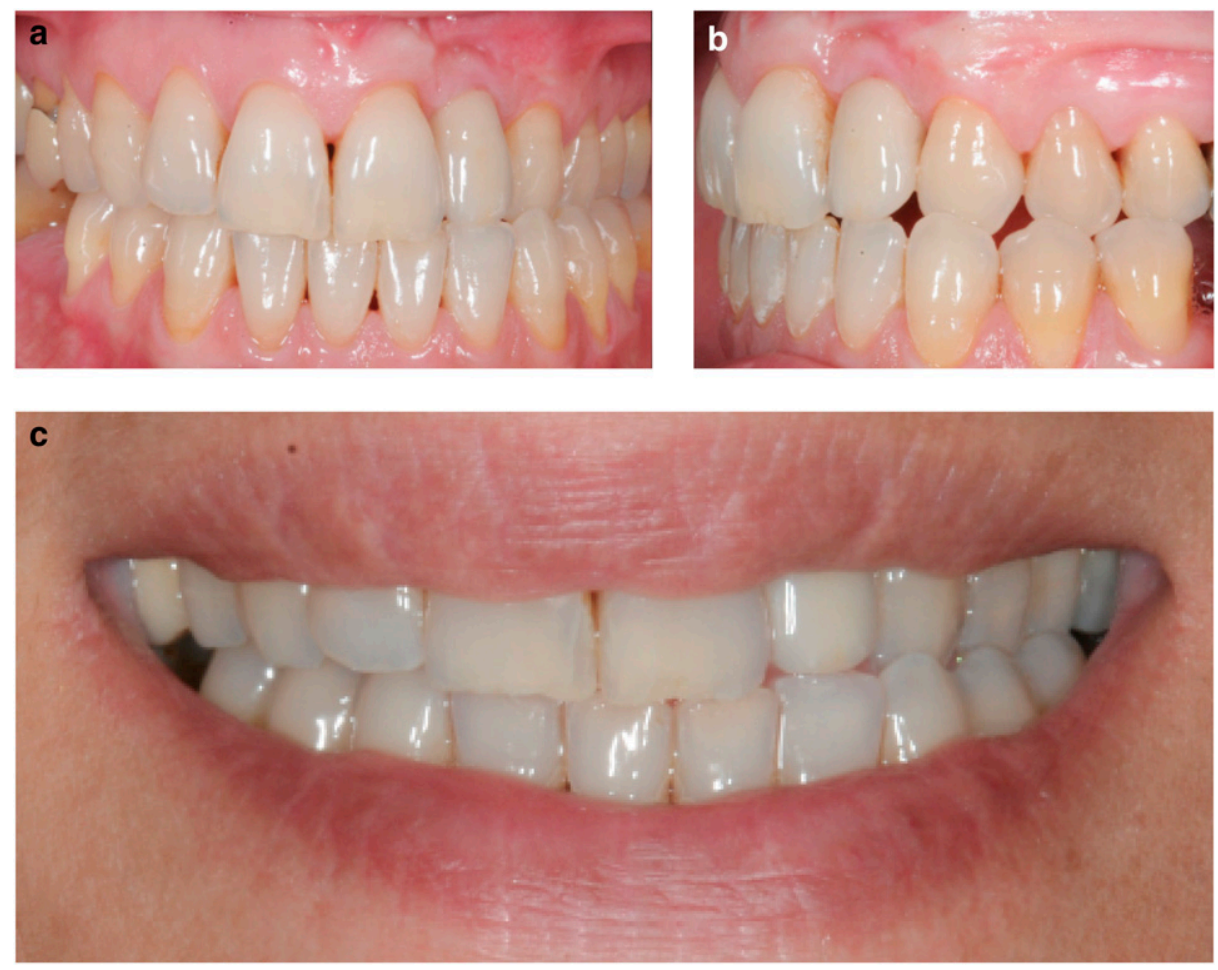

FIGURE 12 Final clinical extraoral views of the restoration. A harmonious gingival margin preserving a convex contour can be observed. 12a Frontal view. 12b Lateral view. 12c Smile after restoration.

on top of the membrane was to promote wound healing as platelets aggregate and release cytokines, growth factors, and hemostatic factors during the first phase of wound healing. ${ }^{12}$
An autogenous block graft, xenogenic particulated graft, and absorbable collagen plus PRP were successfully incorporated in augmenting a significant deficient horizontal and vertical ridge in the maxillary anterior region.

\section{Summary}

Why is this case new information?

- Ramus block grafts placed on both buccal and palatal sides in the maxillary anterior region to augment a horizontally and vertically deficient ridge

- Vertical augmentation achieved with a ramus block graft

What are the keys to successful management of this case?
Proper treatment plan

- Carefully executed surgical plan, which follows the "PASS" principle, ${ }^{13}$ the key to achieving predictable bone regeneration

- Understand the biologic properties of each material used

- Ensure that there is no mobility of the block graft and no dead space between the graft and the recipient bed
What are the primary limitations to success in this case?
- Lack of primary wound coverage

- Lack of graft stability

- Poor oral hygiene and premature loading

\section{Acknowledgments}

This case report was financially supported by the Foundation for the Study of Implantology, Oral and Maxillofacial Surgery, Badajoz, Spain. The authors thank Dr. Jia-Hui $\mathrm{Fu}$, Department of Periodontics, Faculty of Dentistry,
National University of Singapore, Singapore, for editing this manuscript. The authors report no conflicts of interest related to this case report.

CORRESPONDENCE:

Dr. Alberto Monje, Juan Miró 16-17, 06011 Badajoz, Spain. E-mail: amonjec@umich.edu. 


\section{References}

1. McCarthy C, Patel RR, Wragg PF, Brook IM. Dental implants and onlay bone grafts in the anterior maxilla: Analysis of clinical outcome. Int J Oral Maxillofac Implants 2003;18:238-241.

2. Garber DA, Rosenberg ES. The edentulous ridge in fixed prosthodontics. Compend Contin Educ Dent 1981;2:212-223.

3. Zins JE, Whitaker LA. Membranous versus endochondral bone: Implications for craniofacial reconstruction. Plast Reconstr Surg 1983;72:778-785.

4. Schwartz-Arad D, Levin L. Intraoral autogenous block onlay bone grafting for extensive reconstruction of atrophic maxillary alveolar ridges. J Periodontol 2005;76:636-641.

5. Felice P, Pistilli R, Lizio G, Pellegrino G, Nisii A, Marchetti C. Inlay versus onlay iliac bone grafting in atrophic posterior mandible: A prospective controlled clinical trial for the comparison of two techniques. Clin Implant Dent Relat Res 2009;11(Suppl. 1):e69-e82.

6. Huang LH, Neiva RE, Wang HL. Factors affecting the outcomes of coronally advanced flap root coverage procedure. J Periodontol 2005; 76:1729-1734

indicates key references.
7. McAllister BS, Haghighat K. Bone augmentation techniques. J Periodontol 2007;78:377-396.

8. Doblin JM, Salkin LM, Mellado JR, Freedman AL, Stein MD. A histologic evaluation of localized ridge augmentation utilizing DFDBA in combination with e-PTFE membranes and stainless steel bone pins in humans. Int J Periodontics Restorative Dent 1996;16:120-129.

9. Marinucci L, Lilli C, Baroni T, et al. In vitro comparison of bioabsorbable and non-resorbable membranes in bone regeneration. J Periodontol 2001;72:753-759.

10. Proussaefs P, Lozada J. The use of intraorally harvested autogenous block grafts for vertical alveolar ridge augmentation: A human study. Int J Periodontics Restorative Dent 2005;25:351-363.

11. Eppley BL, Woodell JE, Higgins J. Platelet quantification and growth factor analysis from platelet-rich plasma: Implications for wound healing. Plast Reconstr Surg 2004;114:1502-1508.

12. Roukis TS, Zgonis T, Tiernan B. Autologous platelet-rich plasma for wound and osseous healing: A review of the literature and commercially available products. Adv Ther 2006;23:218-237.

13. Wang HL, Boyapati L. "PASS" principles for predictable bone regeneration. Implant Dent 2006;15:8-17. 